Ges. Winterthur ${ }_{77} /{ }_{1} 8$ (1927-1930), als Diss. 1928. - (1934): Zur Glazialgeologie des Glattales Eclogae geol. Helv. 27i1: 33-43. - (1946): Exkursion Nr. 13: Uster-Wetzikon-Dürnten-BäretswilStoffel-Hittnau-Pfäffikon - In: Geologische Exkursionen in der Umgebung von Zürich - Geol. Ges. Zürich, Zürich (Leemann). Weber, J. (1901): Beiträge zur Geologie der Umgebung des Pfäffikersees - Mitt. naturw. Ges. Winterthur 3. - (1924): Erläuterungen zur geologischen Karte der Umgebung von Winterthur 1:25000 - Geol. Karte Schweiz 2I u. Mitt. naturw. Ges. Winterthur 15. Wegelin, H. und Gubler, E. (1928): Deckenschotter auf der Heid - Mitt. thurg. naturf. Ges. 27. Wetrstein, A. (1885): Geologie von Zürich und Umgebung, mit geol. Karte 1:40000 Diss. Univ. Zürich, Frauenfeld (Huber). Woldstedr, P. (1958): Das Eiszeitalter. Grundlinien einer Geologie des Quartärs, 2 - Stuttgart (Enke).

\title{
LOCALITÉS SUISSES TIRANT LEUR ORIGINE DE DOMAINES GALLO-ROMAINS Maurice-Ed. Perret
}

Jules César, dans ses Commentaires de la guerre des Gaules, raconte la tentative d'émigration des Helvètes. Ceux-ci, battus par les Romains, durent rentrer dans leur pays et rebâtir les douze villes et les quatre cents villages qu'ils avaient incendiés avant de partir. Nulle part ne figure la liste des douze villes et l'on est réduit à des hypothèses sur leur identification; quant aux villages, on n'en sait presque rien. L'on n'a pas de détails sur la manière dont les Romains occupèrent le pays. Dans toute la Gaule, ils créèrent des domaines agricoles, au centre de chacun desquels s'élevait un groupe de bâtiments, la villa. Les villas primitives se sont généralement agrandies et sont devenues des villages. Lorsque le pays a été christianisé, c'est auprès de la villa que l'on a construit une chapelle et ainsi nombre de villas sont devenues des communes et des paroisses.

Le domaine gallo-romain, en latin fundus, était désigné par un adjectif dérivé du nom de famille romain (gentilice) ou d'un nom d'individu romain ou gaulois. Ainsi le domaine d'une famille Colonius se nommait fundus coloniacus, simplifié en Coloniacum, aujourd'hui Cologny. Par suite de l'évolution de la langue, la terminaison -acum a suivi un sort différent selon les régions de la Gaule. En Suisse romande, après avoir pris au moyen âge la forme -ie, elle est devenue soit $-y$ (Montagny de Montanius), -ex (Saconnex de Saconnus), -ez (Chevenez de Cavinus), -er (Auvernier de Avernius), plus rarement -ay (Chavornay de Cavorinus), -ey (Chabrey de Cabrus); dans les régions alémaniques -acum est devenu -ach (Alpnach de Alpinus).

Le fait que le nom d'une localité se termine par $-y,-e x,-e z,-e r,-a y,-e y$ ou $-a c h$, n'est pas suffisant pour être une preuve certaine d'origine gallo-romaine. Beaucoup de noms terminés ainsi sont moins anciens; par exemple $A c h$ en allemand ancien désignait un cours d'eau et la plupart des noms où l'on retrouve cette terminaison n'ont pas de relation avec les Gallo-Romains. On peut cependant trouver d'autres preuves. Tout d'abord, des fouilles archéologiques ont pu mettre à jour des vestiges de villa galloromaine ou simplement des objets d'époque romaine; ainsi les restes d'une importante villa à Alpnach. Dans certains cas, des documents du moyen âge indiquent des formes anciennes de localités dans lesquelles on reconnaît sans hésitation un nom romain et la terminaison -acum. Dans certains noms modernes, on peut facilement déceler un nom de famille ou d'individu, romain ou gaulois qui se retrouve dans d'autres noms de lieux; certains gentilices étaient très répandus, ainsi Florius est reconnaissable dans les noms de plus de cinquante localités de l'ancienne Gaule, c'est de lui que vient chez nous Fleurier. Enfin, la situation géographique permet l'identification de nombreux domaines gallo-romains: en effet, les villas romaines n'ont pas été placées au hasard; les villages actuels qui leur ont succédé sont habituellement dans de belles situations, bien exposés au soleil, entourés de champs fertiles; on ne les trouve pas dans les bas-fonds sujets aux brouillards, aux inversions de température, aux gelées d'hiver, ni près des marais, ni au bord des lacs ou des rivières qui peuvent déborder, ni sur les crêtes balayées par les vents, ni sur des pentes escarpées, mais, partout où cela est possible, sur un replat, sur une petite éminence au-dessus de la plaine, du lac ou du fond de la vallée; enfin, elles sont situées en général dans le voisinage des villes gallo-romaines ou à proximité de routes romaines. 


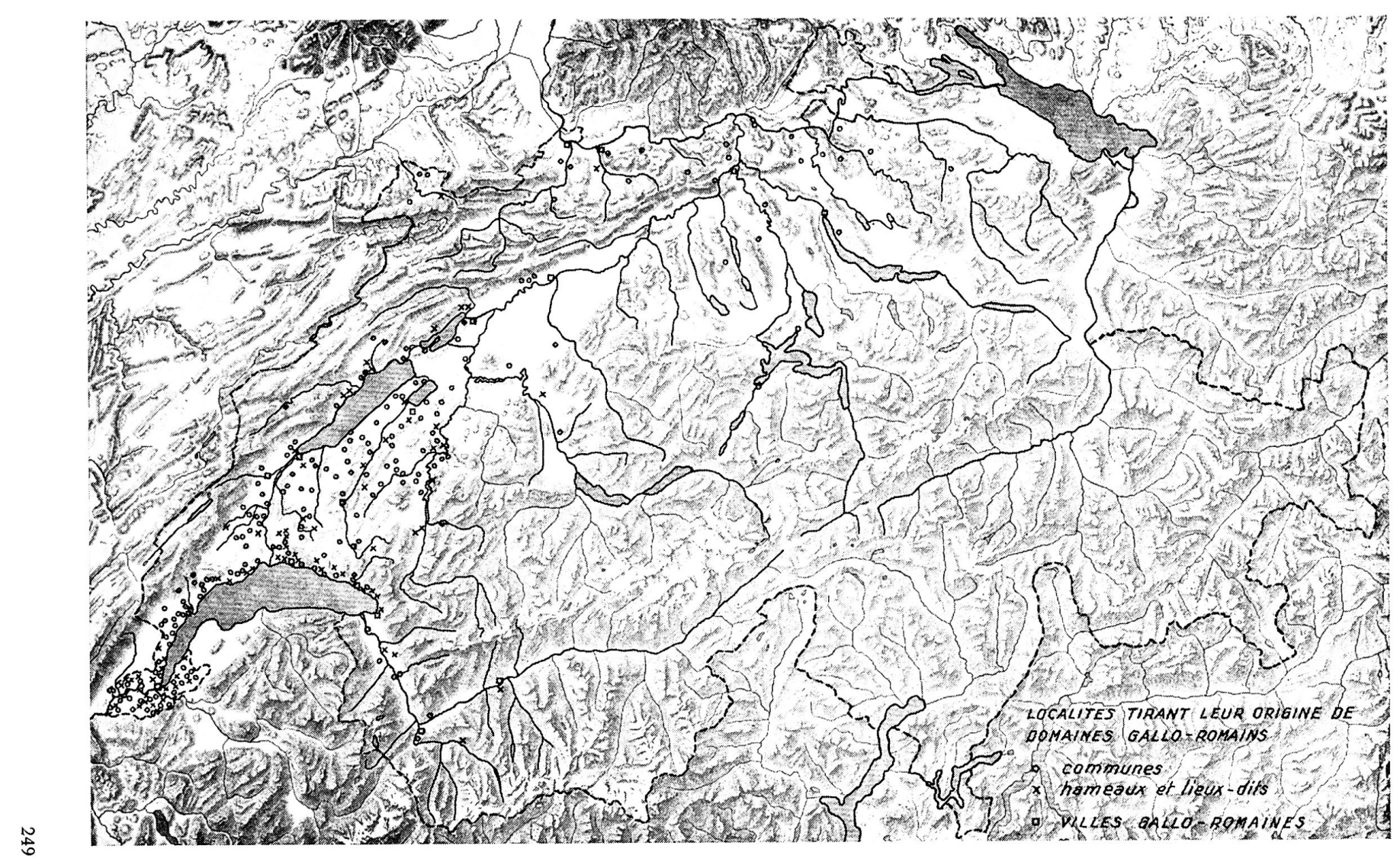


En Suisse, ce ne sont pas moins de cent quatre-vingt-cinq communes dont le nom dérive de la désignation régulière des domaines gallo-romains, c'est-à-dire d'un nom avec la terminaison latine -acum. C'est le canton de Genève qui accuse la plus forte proportion: vingt-six sur le total de quarante-cinq communes; Vaud, Fribourg, Neuchâtel en ont un assez grand nombre; on en rencontre quelques-unes en Valais, dans le Jura bernois et en Suisse alémanique, surtout dans la partie septentrionale de Bâle à la Thurgovie. Ce ne sont pas toutes les communes qui tirent leur origine de domaines gallo-romains. Il est arrivé que des noms aient été remplacés par d'autres: parfois le nom du patron de l'église s'est substitué au nom antique pour désigner la commune, ainsi Saint-Prex, dont trois quartiers, Agny, Marcy et Drassy indiquent trois domaines gallo-romains; la commune de Saint-Barthélemy s'appelait encore en 1860 Bretigny sur Saint-Barthélemy; Saint-Saphorin a probablement remplacé le nom d'un domaine, car on y a retrouvé les vestiges d'une villa. Il en est de même de Saint-Cierges. Il est en outre possible que certains domaines aient eu un nom non pas avec le suffixe-acum, mais avec un autre suffixe. Ce pourrait être notamment le cas des noms en -inges de la région de Genève, par exemple Presinges dans lequel on reconnaît le gentilice romain Priscianus, Puplinges provenant de Popillius; ou les noms en -ins des environs de Noviodunum (Nyon): Prangins de Primius, Begnins de Bennius, Givrins de Gabrius.

Tous les domaines gallo-romains ne sont pas devenus des communes. Un certain nombre sont restés des hameaux ou de simples fermes isolées. Le nom de quelques-uns subsiste dans des lieux-dits désignant des champs ou des bois où l'on a souvent découvert des traces de villas romaines probablement ruinées par les Barbares. Certains noms enfin se sont complètement perdus, les domaines qu'ils désignaient ont été abandonnés lors des invasions barbares, la forêt a envahi les champs laissés en friche et l'on fait parfois, au-dessous des arbres, des trouvailles d'objets d'époque romaine, des restes de constructions. On rencontre plus de cent quatre-vingt hameaux, maisons isolées ou lieux-dits dont les noms semblent dérivés de domaines gallo-romains; trente-deux sont situés dans le canton de Genève, quatre-vingt-quatre dans le canton de Vaud, cinquante dans le canton de Fribourg.

La carte ci-contre montre toutes les communes qui tirent leur origine de noms en -acum, ainsi qu'un certain nombre de hameaux et lieux-dits, la plupart de ceux dont l'identification est la moins contestable; toutefois, dans le canton de Genève, et sur les coteaux le long du lac Léman jusqu'à Villeneuve, le manque de place n'a pas permis de les signaler tous.

La répartition des domaines gallo-romains est intéressante. En Suisse romande, autour du lac Léman, entre les Préalpes et le Jura jusqu'à la Sarine et en Ajoie, elle doit représenter assez bien le degré de mise en valeur du pays à l'époque romaine; à peu près tous les endroits les plus favorables sont occupés et les trouvailles archéologiques confirment cette opinion. On peut s'étonner, par contre d'en trouver si peu en Valais et outre-Sarine. Le Valais qui, comme les trouvailles archéologiques l'ont montré, devait déjà être peuplé dès l'époque néolithique, n'a probablement pas fait l'objet d'une colonisation romaine importante, les indigènes ont continué à vivre selon leurs coutumes, sans que leurs terres soient réparties en domaines, à l'exception toutefois d'un ou deux domaines voisins des villes: dans Fully ou reconnait le gentilice Follius et dans Martigny Martinus. En Suisse alémanique, par contre, une grande partie du pays a dû être mise en valeur à l'époque romaine, comme le prouvent les nombreuses trouvailles d'époque romaine faites dans des régions qui n'avaient pas fourni de vestiges plus anciens. Il est possible qu'il y ait eu de nombreux domaines gallo-romains qui aient été ruinés lors des invasions barbares, ou bien que les successeurs des Gallo-Romains aient remplacé les noms d'origine latine par des noms germaniques, ou qu'il y ait encore une autre explication. Des études plus poussées permettront peut-être de faire de la lumière sur ce point. 


\section{SCHWEIZERISCHE ORTSCHAFTEN RÖMISCHER HERKUNFT}

Nachdem die Römer ihre Herrschaft über ganz Gallien ausgedehnt hatten, wiesen sie einzelnen Bürgern Grundstücke (fundi) als Eigentum zu. Jedes Grundstück enthielt die Wohnung des Besitzers, die mit den Wirtschaftsgebäuden einen Hof bildete. Die meisten Höfe erweiterten sich später zu Weilern, Dörfern oder Gemeinden. Die Gutshöfe wurden nach dem Namen des ersten Besitzers mit der keltischen Endung -acum bezeichnet. Diese Endung entwickelte je nach den Sprachgebieten verschieden. In der Westschweiz wurde sie zuerst zu -ie, dann zu -y, -ex, -ez, -er und sogar zu -ay, -ez, oder -ey, in der deutschen Schweiz zu -ach. Diese Rückschlüsse auf den Ursprung vieler Siedlungen erlaubenden Ausbildungen werden im vorstehenden Aufsatz diskutiert und kartographisch belegt.

\section{DER PLAN DER STADT BERN UND DES BREMGARTENWALDES VON I623 Georges Grosjean}

Das Bernische Historische Museum birgt eine bisher in der Öffentlichkeit wenig bekannte kartographische Kostbarkeit. Es handelt sich um das Oelgemälde «Grundry $\beta$ der Statt Bern sampt dero selben oben us nechst gelegnen ortten», geschmückt mit schöner Wappenkartusche und dem Bildnis des Seckelmeisters Antoni von Graffenried. Das Porträt trägt die Datierung «Anno 1623». Ohne Rahmen mißt die Karte $79 \times 113,5 \mathrm{~cm}$ und stellt die Gegend der Stadt Bern im Umkreis Untertorbrücke Worblaufen - Reichenbach - Eymatt - Bümpliz - Köniz dar. Die Orientierung ist ungefähr SSE, der Maßstab, nach dem auf dem Plan eingezeichneten Maßstab in Schritten und Ruten 1:6286, variiert in Wirklichkeit von ca. 1:6300 bis ca. 1:8900; im Mittel beträgt er ca. 1:7000.1

Angaben über den Autor enthält die Karte keine. Eine eingehende Untersuchung ergab indessen, $d a ß$ als Autor mit größter Wahrscheinlichkeit JosEPH PLEPP, der bernische Baumeister und Künstler (ein Sohn des Churer Glasmalers H. J. Plepp) anzusprechen ist, was dem Plan eine gewisse Bedeutung verleiht. PLepp's Autorschaft geht u. a. aus einer Eintragung in der Seckelmeisterrechnung des auf unserem Plan dargestellten Antoni von Graffenried hervor: «Joseph Bleppen 37 cronen usgricht, so meine herren die Venner, ime wegen gemachtem Abriß und Grundlags des Bremgartens zu einer Verehrung geschöpfft...» (19. Buch, vom Stephanstag 1622 bis St. Johannstag 1623 , unter «Das allgmein täglich ussgeben», 13. März 1623). Daß der Plan vornehmlich wegen des Bremgartenwaldes aufgenommen wurde, geht auch aus dem obern Textschild des Plans selbst hervor.

Die nach dem Geschmack der barocken Zeit mit reichem Schmuck umrahmten Schriftfelder sind für die Beurteilung unseres Plans von großer Bedeutung. Wir vernehmen nämlich, da $B$ er nicht wie bisher ungenau mit Schritten, sondern mit Meßstangen (Ruten) ausgemessen wurde. Die große und auffällig abgebildete Bussole dürfte nicht nur die Nordrichtung angeben, sondern weist wohl darauf hin, daß eigentliche Bussolenzüge gemacht wurden.

Wir stehen damit vor dem ersten Werk bernischer Kartographie, das mit exakten Methoden aufgenommen wurde. Das Ergebnis ist entsprechend: Die recht schwer aufzunehmenden Aareschleifen der Berner Landschaft sind für die damalige Zeit ganz hervorragend wiedergegeben wie der Vergleich mit der neuen Landeskarte in der beigegebenen Skizze zeigt. Der für die Reduktion angenommene Maßstab wurde so gewählt, daß sich vor allem die östlichen, wichtigeren Teile unseres Plans mit der Landeskarte zur Deckung bringen ließen. Wenn sich die westlichen Teile nur schlecht decken, so ist es, weil die Stadt und eine gewisse mittlere Partie unseres Plans maßstäblich zu groß geraten sind und außerdem die Längsachse der Stadt eine Abweichung von $15^{\mathrm{g}}$ nach Norden in bezug auf die wirkliche Richtung zeigt. An sich ließen sich

1 Ein größerer Ausschnitt $79 \times 67 \mathrm{~cm}$, enthaltend die wichtigeren östlichen Teile, wurde mit freundlicher Erlaubnis des Bernischen Historischen Museums durch den Verlag Kümmerly \& Frey, faksimile reproduziert. Die Mitglieder der Geographisch-Ethnographischen Gesellschaft Zürich, die das Werk unterstützte, erhalten die Reproduktion ungefalzt unentgeltlich gegen Einsendung der beiliegenden Bestellkarte an Firma Kümmerly \& Frey, Bern. Weitere Interessenten können den Plan samt eingehendem Kommentar zum Preise von Fr. 12.5o (plus Porto und Verpackung) bei Kümmerly \& Frey, Bern oder im Buchhandel beziehen. 